journal club

Neuer selektiver S1P1-Rezeptormodulator bei schubförmiger Multipler Sklerose

\title{
Amiselimod in der MOMENTUM-Studie
}

Fragestellung: Ist Amiselimod, ein selektiver Modulator des Sphingosin-1-Phosphat-1(S1P1)-Rezeptors in der Behandlung der schubförmigen Multiplen Sklerose (MS) wirksam?

Hintergrund: Mit Fingolimod wurde vor gut fünf Jahren der erste S1P-Rezeptormodulator für die Therapie der schubförmigen MS zugelassen, nachdem mehrere Phase-III-Studien eine überzeugende Wirksamkeit und Sicherheit nachweisen konnten. Inzwischen wurden weltweit mehr als 150.000 Patienten mit der Substanz behandelt. Bei Patienten mit sekundär chronisch progredienter MS konnte kürzlich ferner gezeigt werden, dass der selektive S1P1-Rezeptormodulator Siponimod die Krankheitsaktivität signifikant reduziert. Mit Amiselimod wurde nun ein weiterer selektiver S1P1-Rezeptormodulator in der Therapie der schubförmigen MS untersucht.

Patienten und Methodik: In dieser doppelblinden, randomisierten placebokontrollierten Phase-II-Studie wurden Patienten mit schubförmiger MS im Alter zwischen 18 und 60 Jahren im Verhältnis von $1: 1: 1: 1$ in vier Gruppen randomisiert. Amiselimod wurde in Dosierungen von $0,1 \mathrm{mg}, 0,2 \mathrm{mg}$ und $0,4 \mathrm{mg}$ versus Placebo untersucht. Der primäre Endpunkt war

Kappos L, Arnold D, Bar-Or A et al. Safety and efficacy of amiselimod in relapsing remitting multiple sclerosis (MOMENTUM): a randomised, double-blind placebo-controlled phase 2 trial.

Lancet Neurol 2016; 15: 1148-59 die Gesamtzahl der Gadolinium aufnehmenden Herde in monatlichen MRT-Untersuchungen in den Wochen 8 bis 24 nach Therapiebeginn. Sekundäre Endpunkte waren weitere MRT-Parameter wie der Anteil der Patienten ohne Gadolinium aufnehmende Herde, die Zahl und das Volumen neuer oder vergrößernder T2-gewichteter Herde in den Wochen 4 bis 24 und klinische Parameter wie die jährliche Schubrate, die Zeit bis zum ersten bestätigten Schub, der Anteil der schubfreien Patienten in Woche 24 sowie verschiedene Progressionsparameter.

Ergebnisse: Zwischen Januar und Dezember 2013 konnten insgesamt 536 Patienten in die Studie eingeschlossen werden, von denen 415 randomisiert wurden. Die Zahl der Gadolinium aufnehmenden Herde in den Wochen 8 bis 24 lag in der Placebogruppe bei 8,3, in der Gruppe mit Amiselimod 0,1 mg bei 5,5 mit Amiselimod 0,2 mg bei 2,3 und mit Amselimod 0,4 mg bei 1,7. Der Unterschied zwischen Placebo und Amiselimod 0,1 mg war nicht signifikant, allerdings hoch signifikant für die beiden höheren Dosisgruppen. Ähnliche Ergebnisse zeigten sich für die anderen MRT-Parameter. Die Zahl der Schübe war nur zwischen Placebo und Amiselimod 0,4 mg signifikant unterschiedlich, die Zeit bis zum ersten Schub zwischen Amiselimod 0,2 mg sowie $0,4 \mathrm{mg}$ und Placebo.

Die Nebenwirkungsrate war bei allen Amiselimod-Gruppen vergleichbar zu Placebo. Schwere Nebenwirkungen traten nicht auf, die klassischen Reduktionen der Herzfrequenz bei Erstgabe waren hier ebenfalls nicht zu beobachten.

Schlussfolgerungen: Amiselimod zeigte in den Dosierungen von $0,2 \mathrm{mg}$ und $0,4 \mathrm{mg}$ signifikante dosisabhängige Wirksamkeiten bei den MRT- sowie den klinischen Parametern. Alle Dosierungen zeigten ein sicheres Nebenwirkungsprofil bei guter Verträglichkeit.

\section{- Kommentar von Volker Limmroth, Köln}

\section{Noch ein selektiver S1P1-Rezeptormodulator ...}

Trotz der überzeugenden Wirksamkeit und des guten Nebenwirkungsprofils wird man sich den Namen Amiselimod wahrscheinlich nicht merken müssen, denn die Substanz wird wohl nicht weiterentwickelt - offensichtlich nicht aus medizinischen Gründen. Warum ist die Studie trotzdem interessant? 1. An der guten Wirksamkeit der S1P1-Modulation in der Therapie der schubförmigen MS dürfte nun wirklich kein Zweifel mehr bestehen. 2. Im Vergleich zur nicht selektiven S1P-Modulation scheint die selektive S1P1-Modulation tatsächlich einige kleine Vorteile zu haben. Ob die selektiven Substanzen auch wirksamer sind, kann zwar noch nicht sicher beurteilt werden (und wenn, dann sind die Unterschiede gering), aber das Nebenwirkungsspektrum scheint unter der selektiven S1P1-Modulation etwas vorteilhafter. Die Tatsache, dass die Nebenwirkungsrate aller Dosierungen (auch der höheren) mit Placebo vergleichbar war, ist bemerkenswert. Ebenso zeigt die fehlende Beeinflussung der Herzfrequenz, dass das kardiologische Profil durch die selektive S1P1-Modulation offensichtlich verbessert wird. Aller- dings muss hier berücksichtigt werden, dass das Sicherheitsprofil von Fingolimod bereits vergleichsweise gut ist. Und ob diese kleinen Unterschiede zwischen einer gut etablierten Substanz wie Fingolimod, die in absehbarer Zeit bereits generisch wird, und einer neuen Substanz, die noch durch die Phase III muss, die Entwicklungskosten wert sind, entscheiden im Zweifelsfall nicht die Mediziner in der Entwicklungsabteilung.

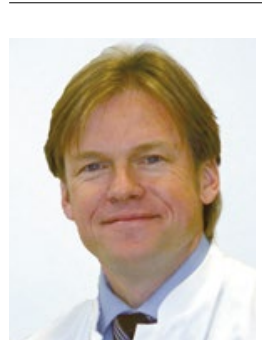

Prof. Dr. med. Volker Limmroth, Köln-Merheim

Chefarzt der Klinik für Neurologie und Palliativmedizin Köln-Merheim E-Mail: LimmrothV@kliniken-koeln.de 